podium is cut, the proximal part is rapidly withdrawn. Sometimes this takes place rather violently, and the pseudopodium is then thrown into loose spiral coils in the process. In the part distal to the cut the streaming movements continue unchanged for some time; but gradually the movement becomes preponderatingly towards the cut, and protoplasm accumulates there in the form of a swollen mass. Little or no movement can be seen in this mass, which is therefore probably more solid than the ordinary pseudopodial protoplasm. Subsequent slow general contraction of the whole of the pseudopodial reticulum distal to the cut results in the withdrawal of this part away from the place of the cut; but usually before this contraction is complete new pseudopodia begin to grow out from various parts of this now enucleated fragment. I have no record of coiling ever occurring during this slow contraction.

Thus, in contrast to the cut nerve fibre, in a cut pseudopodium it is the part connected with the nucleus which shrinks, while the swelling occurs at the distal side of the cut. Coiling only occurs in the nucleated fragment, and then only if the retraction is sudden. The fact that new pseudopodia grow from the enucleated fragment shows that contact with the nucleus is not required in order to orientate the micellæ or chain molecules, the existence of which has to be assumed in order to account for the structure and movements of the pseudopodia.

The weakness of Verworn's analogy lies largely in the fact that the pseudopodium is not to any extent specialized for the purpose of conduction. A stimulus given to one part of the pseudopodial reticulum has no effect on adjacent parts unless it involves a gross mechanical disturbance or a change in the streaming movements sufficient to extend to those parts. Nevertheless, from the point of view of the conditions necessary for maintaining physical stability in fine elongated strands of protoplasm, the larger Foraminifera provide particularly attractive material for study.

Zoological Department,

H. Sandon. Rondebosch,

University of Cape Town.

1 Verworn, M., "Irritability" (Yale Univ:_Press, 1913).

"Young, J. Z., Nature, 153, 333 (1944).

'Lepeshkin, W. W., Biologia Generalis, 1, 368 (1925).

'Sandon, H., Nature, 133, 761 (1934).

\section{Energy Imparted by Active Nitrogen}

Spectroscopic evidence ${ }^{1}$ shows that the maximum energy of excitation which a molecule of active nitrogen can impart to another molecule (or atom) is $9 \cdot 45 \mathrm{eV}$. Lord Rayleigh, however, from a study of the incandescence of metals immersed in active nitrogen, finds ${ }^{2}$ that each molecule of active nitrogen delivers to the metal energy of, at the least, $10 \mathrm{eV}$. These apparently conflicting results can be reconciled and satisfactorily explained on the hypothesis recently proposed by me, namely, that active nitrogen is simply the ionized molecule of nitrogen $\mathrm{N}_{2}+\left(X^{\prime}\right)$ produced by the discharge .

The molecules (or atoms) introduced into the vessel containing active nitrogen are excited by a three-body collision process as follows :

$\mathrm{N}_{2}++e+M \rightarrow \mathrm{N}_{2}$ (excited) $+M$ (excited).

Now, the lowest electronic level of excitation to which $\mathrm{N}_{2}$ can drop, on neutralization, is the $A$-level, with energy $6 \cdot 1 \mathrm{eV}$. The levels lying immediately below it are high vibrational levels of the ground state $(X)$ with distances of nuclear turning-points very different from the nuclear separation of $\mathrm{N}_{2}\left(X^{\prime}\right)$. Transitions to any of these levels will violate the Franck-Condon principle. The maximum energy left over for exciting the third body is thus $15 \cdot 58-6 \cdot 1=9 \cdot 48 \mathrm{eV}$. This explains why repeated attempts by spectroscopists have failed to produce excitation levels above $9 \cdot 45 \mathrm{eV}$.

The possible levels below the $A$-level to which the neutralized $\mathrm{N}_{2}$ molecule can drop are the ground-level $(X)$ and a few of the vibration-levels immediately above it. But this would mean that nearly the whole of the energy of ionization is either radiated away or is taken up by $M$. The reaction in this case would be :

$\mathrm{N}_{2}++e+M \rightarrow \mathrm{N}_{2}$ (normal state) $+M$ (excited state). I do not know if the probability of such reactions has been studied. In my opinion the probability would be very small.

To explain the higher value of energy as obtained by Rayleigh we recall that for neutralization of $\mathrm{N}_{2}+$ on the surface of a solid, the latter acts as the third body. The electrons first arrive on the surface of the solid and remain there as surface charge. The $\mathrm{N}_{2}+$ ions then arrive and combine with the electrons, giving up the energy of recombination to the solid surface. Since the solid, with its complicated structure, has many modes of vibration, it can take up the whole of the released energy, $15 \cdot 58 \mathrm{eV}$. This, in other words, means that though spectroscopically active nitrogen can impart energy only up to a maximum of $9 \cdot 45 \mathrm{eV}$. to an atom or a molecule, it can impart much greater energy $(15 \cdot 58 \mathrm{eV}$.) to the surface of a solid. This explains the apparently conflicting results mentioned above.

c/o Royal Society,

S. K. Mitra.

London.

Nov. 10.

1 Okubo, J., and Hamada, H., Phil. Mag., (7), 5, 272 (1928).

${ }^{2}$ Rayleigh, Lord, Proe. Roy. Soc., A, 176, 17 (1940).

${ }^{3}$ Mitra, S. K., Science and Culture (Calcutta), 9, 49 (1942-43) : 10 $133(1944-45) ;$ Nature, 154, 212 and 576 (1944).

\section{Permeability of Adsorbing Substances}

KING has shown ${ }^{1}$ that at low concentrations the diffusion constant of water in keratin becomes extremely small in comparison with its value at higher concentrations. This effect is already known from the behaviour of the hair hygrometer ${ }^{2}$, the response of which (dependent on the internal diffusion of water in keratin) is much slower at low than at high relative humidities. The magnitude of this effect is shown by the following figures I have obtained for the times $(\tau)$ of half-change of the length of hair (of $0 \cdot 1 \mathrm{~mm}$. diameter) after a sudden small change of humidity, at $18^{\circ} \mathrm{C}$.

Per cent
relative humidity
$10-25$
$25-34$
$34-49$
$49-63$
$63-75$
$75-92$

$\tau$ (sec.)
150
65
40
26
15
$<10$

That this phenomenon is characteristic not only of keratins but also of other adsorbing substances, for example cellulose, can be shown by a very simple experiment, reminiscent of King's', but more suit- 